\title{
Metabolic engineering of Methylobacterium extorquens AM1 for 1-butanol production
}

Bo $\mathrm{Hu}^{1 *}$ and Mary E Lidstrom ${ }^{1,2}$

\begin{abstract}
Background: Butanol is a promising next generation fuel and a bulk chemical precursor. Although clostridia are the primary industrial microbes for the fermentative production of 1-butanol, alternative engineered hosts have the potential to generate 1-butanol from alternative carbon feedstocks via synthetic metabolic pathways. Methylobacterium extorquens AM1, a facultative methylotrophic a-proteobacterium, is a model system for assessing the possibility of generating products such as 1-butanol from one-carbon and two-carbon feedstocks. Moreover, the core methylotrophic pathways in $M$. extorquens AM1 involve unusual coenzyme A (CoA)-derivative metabolites, such as crotonyl-CoA, which is a precursor for the production of 1-butanol.

Results: In this work, we engineered a modified CoA-dependent pathway in Methylobacterium extorquens AM1 to produce 1-butanol. Engineered strains displayed different 1-butanol titers using ethylamine as a substrate. A strain overexpressing Treponema denticola trans-enoyl-CoA reductase, Clostridium acetobutylicum alcohol dehydrogenase, and native crotonase was able to generate the highest 1-butanol titer $\left(15.2 \mathrm{mg}^{-1}\right)$. In vitro isotopic tracing of metabolic flux and in vivo metabolite analysis showed the accumulation of butyryl-CoA, demonstrating the functionality of the synthetic pathway and identifying targets for future improvement.

Conclusions: We demonstrated the feasibility of using metabolic intermediates of the ethylmalonyl-CoA pathway in M. extorquens AM1 to generate value-added chemicals, with 1-butanol as the test case. This will not only establish the biotechnological potential of the ethylmalonyl-CoA pathway, but will also introduce M. extorquens AM1 as a potential platform to produce value-added chemicals.
\end{abstract}

Keywords: Methylobacterium extorquens AM1, 1-butanol, Ethylmalonyl-CoA pathway

\section{Background}

1-butanol has been proposed to be a better alternative to ethanol as a replacement for gasoline because of its many advantages [1]. 1-butanol is less soluble in water than ethanol, reducing the possibility of contamination in groundwater, and this trait also makes it feasible to use liquid-liquid extraction for 1-butanol recovery in industrial fermentation. Unlike ethanol, 1-butanol is compatible with the current petroleum infrastructure and can be blended with gasoline in a high ratio [2]. The energy density of 1-butanol is close to that of gasoline, so one gallon of gasoline blended 50:50 (v/v) with 1-butanol produces a similar mileage to that of pure gasoline $[3,4]$.

\footnotetext{
* Correspondence: bohu@uw.edu

${ }^{1}$ Department of Chemical Engineering, University of Washington, Seattle, WA, USA

Full list of author information is available at the end of the article
}

Fermentative production of 1-butanol by clostridial species has a long history as the main industrial 1butanol-producing process [4]. Although recent development of new genetic tools for solventogenic clostridia has led to evolved strains with a combination of desired traits $[5,6]$, industrial fermentation of clostridia still has limitations such as formation of byproducts, spore formation, and low cell density [7]. Therefore, there is growing interest in metabolically engineering alternative hosts for 1-butanol production. Recently, several groups have reported successful reconstruction of the butanol synthesis pathway of clostridia in non-native hosts, including Escherichia coli [8], Saccharomyces cerevisiae [9], Pseudomonas putida, Bacillus subtilis [10], and Lactobacillus brevis [11]. While starchy substrates or molasses are major carbon feedstocks for fermentative production of 1-butanol in clostridia or heterologous microorganisms, interest is growing in employing novel 
substrates that are economically competitive with petrochemical synthesis yet at the same time non-competitive with food for human consumption [12]. In the last few years, attention has focused on single carbon compounds such as methane and methanol as future alternative carbon feedstocks due to their relative abundance [13]. However, 1-butanol has not yet been reported to be produced by organisms capable of growing on methane (methanotrophs) or methanol (methylotrophs).

Methylobacterium extorquens AM1 is a facultative methylotrophic $\alpha$-proteobacterium capable of using both one-carbon (C1) compounds as well as multi-carbon compounds as sole carbon and energy sources. The potential practical significance of $M$. extorquens AM1 in biotechnology, such as in the biosynthesis of amino acids and single-cell proteins and the bioconversion of methanol into products with economic value, has brought it into prominence since the 1960s [14]. As the most well-understood methylotroph, $M$. extorquens AM1 is a potential platform for converting methanol to biofuels, building on the elucidation of pathways involved in $\mathrm{C} 1$ and $\mathrm{C} 2$ metabolism and development of tools for metabolic engineering. The ethylmalonyl-CoA pathway (EMC pathway, Figure 1), a central metabolic pathway in M. extorquens AM1, converts acetyl-CoA to glyoxylate for reincorporation into the serine cycle during $\mathrm{C} 1$ assimilation and replenishes metabolites that either leave the cycle for biosynthetic purposes or are consumed by the tricarboxylic acid (TCA) cycle during C2 assimilation [15,16]. The EMC pathway involves unusual metabolites that are of interest for the production of valuable compounds, such as crotonyl-CoA, a key precursor of the 1-butanol biosynthesis pathway in clostridia (Figure 1). Moreover, it has been shown that significant metabolic flux occurs through the EMC pathway during growth of $M$. extorquens AM1 on either $\mathrm{C} 1$ or $\mathrm{C} 2$ compounds, generating a stable supply of crotonyl-CoA as a direct precursor for 1-butanol production [17]. Therefore, in this work we describe the engineering of a modified CoA-dependent pathway in M. extorquens AM1 to produce 1-butanol.

\section{Results and discussion}

\section{Construction of 1-butanol biosynthesis pathway}

The essential enzymes for 1-butanol production are outlined in Figure 1, three of which (PhaA, PhaB, and CroR) are already present in the EMC pathway of $M$. extorquens AM1. In addition to these existing enzymes, heterologous enzymes were introduced to convert crotonyl-CoA to 1-butanol. These include an enzyme catalyzing the reduction of crotonyl-CoA to butyryl-CoA (Bcd and EtfAB from Clostridium acetobutylicum, or Ter from Treponema denticola) and an alcohol dehydrogenase from Clostridium acetobutylicum that converts butyryl-CoA to 1-butanol $[18,19]$. Two alcohol dehydrogenases are present in Clostridium acetobutylicum: AdhE1 and AdhE2 [20]. Although engineered strains overexpressing either of the homologs produced a similar level of 1-butanol [10], AdhE2 was thought to be the more active homolog [21] and thus was used in this study for the reduction of butyryl-CoA. The enzyme-coding genes were expressed in a polycistronic manner driven by promoters developed for overexpression in $M$. extorquens AM1. Four promoters

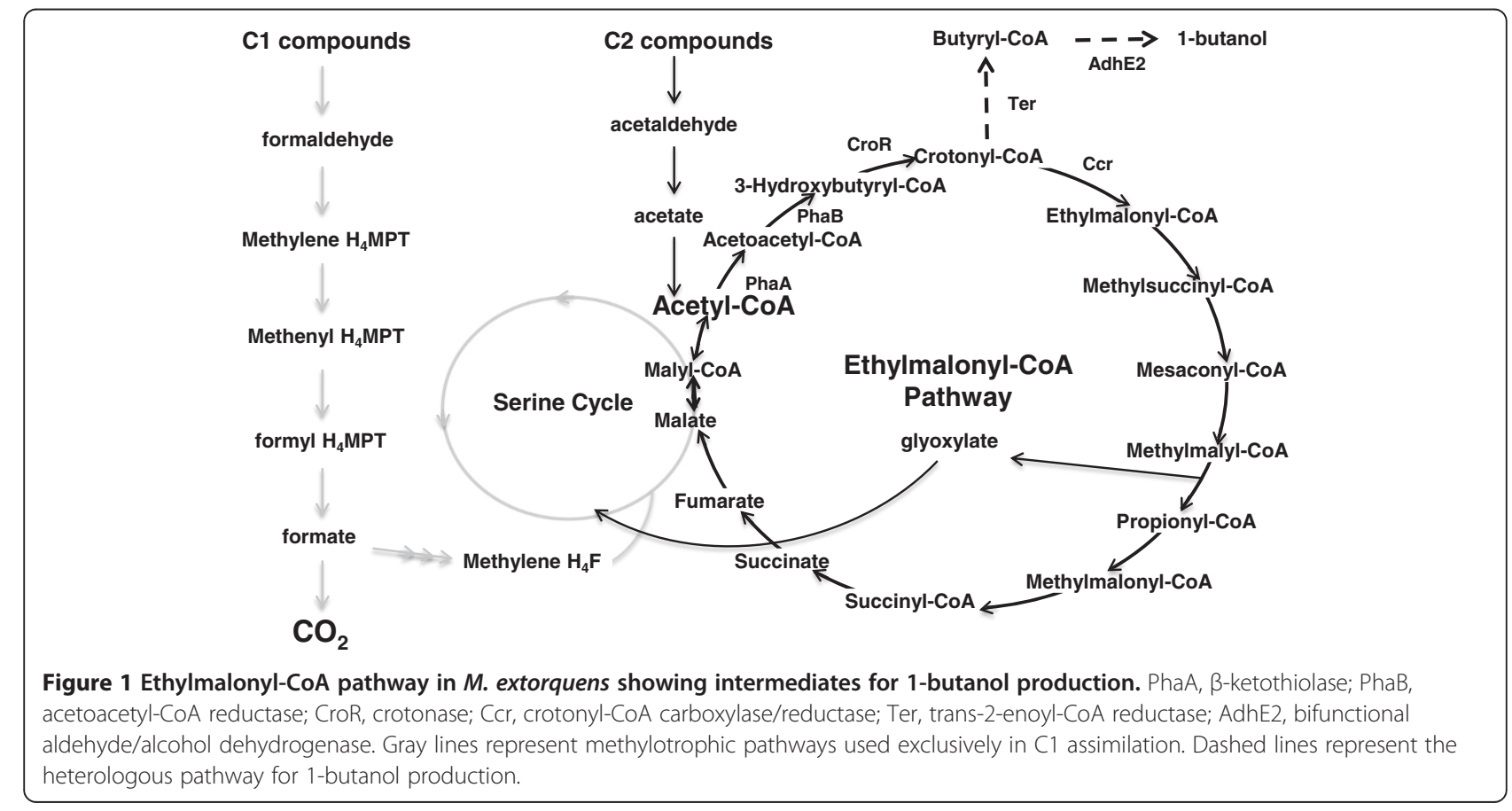


were tested, ranging from high to low expression, including those for mxaF, lac [22], and two chosen based on microarray data [23-25].

Engineered strains were tested for 1-butanol production using methanol or ethylamine as the carbon source. Notably, no methanol-grown strains produced detectable 1-butanol. We hypothesized that the methanol dehydrogenase in $M$. extorquens AM1, a pyrroloquinoline quinone (PQQ)-dependent periplasmic alcohol dehydrogenase that can interconvert $\mathrm{C} 1-\mathrm{C} 4$ alcohols and aldehydes, may interfere with net conversion of butyraldehyde to 1-butanol by catalyzing the reverse reaction in the periplasm [26]. Therefore, we also tested 1-butanol production from a $m x a F$ mutant growing on methylamine, which does not contain significant methanol dehydrogenase activity [27]. However, no 1-butanol was detected, suggesting that endogenous butanol dehydrogenase activity may not be an important factor causing low 1-butanol titer. An alternative explanation is that the glyoxylate generated by the EMC pathway is essential for growth on methanol [28]. Tests for 1-butanol production from cells grown on methanol plus glyoxylate at a concentration known to rescue mutants in the EMC pathway for growth on methanol [29] did not result in detectable 1-butanol production, suggesting that lack of glyoxylate production was not a limiting factor.

Although the constructs tested were unable to produce 1-butanol during growth on methanol, three of the strains (BHB4, BHB7, and BHB8) produce detectable levels of 1 -butanol during growth on ethylamine (Table 1), with

Table $1 M$. extorquens strains and plasmids used in this study

\begin{tabular}{|c|c|c|}
\hline Strain & Plasmid/Genotype* & Reference \\
\hline $\begin{array}{l}\text { Wild } \\
\text { type }\end{array}$ & Rif derivative & 27 \\
\hline BHB1 & pCM66 (Plac:: bcd\&etf-adhE2) & This study \\
\hline BHB2 & pCM80 (PmxaF:: bcd\&etf-adhE2) & This study \\
\hline BHB3 & pAP775 (Pmeta1_3616 :: bcd\&etf-adhE2) & This study \\
\hline BHB4 & pCM80 (PmaxF:: adhE2-ter) & This study \\
\hline BHB5 & pCM66 (Plac:: adhE2-ter) & This study \\
\hline BHB6 & pAP776 (Pmeta1_002:: adhE2-ter) & This study \\
\hline BHB7 & pAP775 (Pmeta1_3616 :: adhE2-ter) & This study \\
\hline BHB8 & pAP775 (Pmeta1_3616 :: ter-adhE2) & This study \\
\hline BHB9 & pHC61 (Pmtac:: croR:: Pmeta1_3616 :: adhE2-ter) & This study \\
\hline Plasmids & Description & Reference \\
\hline pCM66 & M. extorquens expression vector (Plac, $\mathrm{Km}^{\mathrm{R}}$ ) & 25 \\
\hline pCM80 & M. extorquens expression vector $\left(\mathrm{PmxaF}, T c^{R}\right)$ & 25 \\
\hline pAP775 & M. extorquens expression vector (Pmeta1_3616, Tc $c^{R}$ ) & E. Skovran \\
\hline pAP776 & M. extorquens expression vector (Pmeta1_002, TC ${ }^{R}$ ) & E. Skovran \\
\hline pHC61 & M. extorquens expression vector (Pmtac, $\mathrm{Km}^{\mathrm{R}}$ ) & 47 \\
\hline
\end{tabular}

*Promoter strength: PmxaF > Pmeta1_3616 > Pmeta1_002 > Plac.
BHB7 showing the highest titer of $8.9 \mathrm{mg} \mathrm{l}^{-1}$ (Figure 2). The titer is comparable with that of early reported organisms such as $S$. cerevisiae (2.5 $\mathrm{mg} \mathrm{l}^{-1}$, [9]) and cyanobacteria (14.5 $\mathrm{mg} \mathrm{l}^{-1}$, [30]), but comparison of our strain to the native 1-butanol producers Clostridium (about $15 \mathrm{~g} \mathrm{~L}^{-1}$ ) or the engineered $E$. coli strains (about $15 \mathrm{~g} \mathrm{~L}^{-1}$ ) demonstrates the need for significant additional 1-butanol titer improvement [31,32]. Growth on C2 compounds is significantly different from growth on $\mathrm{C} 1$ compounds, which likely underlies the difference in 1-butanol production. During growth on C2 compounds, fewer steps are required for converting $\mathrm{C} 2$ compounds into acetylCoA. It has been suggested that the NADH pool is critical to drive 1-butanol synthesis due to the large NADH consumption by the 1-butanol production pathway [31]. Although neither of the NADH-linked formate dehydrogenases is essential for cell growth [33], a stoichiometric model suggests that biomass synthesis is limited by reducing power rather than ATP during growth on $\mathrm{C} 1$ compounds, but not on C2 compounds [16,34]. Moreover, during growth on two-carbon compounds, the level of NADH is different than that on methanol [16]. One or more of these differences may be the reason why none of these constructs accumulated detectable amounts of 1-butanol during growth on methanol.

The three strains that generated detectable 1-butanol in ethylamine-grown cells, BHB4, BHB7, and BHB8, all contained Ter instead of the Bcd and EtfAB cluster. This is possibly due to the oxygen sensitivity and instability of the Bcd and EtfAB complex [18,35], since $M$. extorquens AM1 is an aerobic methylotroph that does not grow under anoxic conditions. The strain that generates the highest 1-butanol titer (BHB7) expresses the heterologous genes from a promoter of intermediate strength (Pmeta1_3616, [23]), suggesting that tuning of expression levels is critical for proper functioning of the 1-butanol production pathway.

\section{Enzyme activities}

Activities of the heterologous enzymes of the 1-butanol pathway were measured in extracts of cells grown on ethylamine. Both butyraldehyde dehydrogenase activity and butanol dehydrogenase activity of AdhE2 were detected above the background activity present in wild-type M. extorquens AM1 (Table 2). Attempts to measure a combined Bcd and EtfAB activity were not successful using crude extract from cells that expressed both $b c d$ and etfAB. The lack of detectable activity may be due to the high oxygen sensitivity of the Bcd and EtfAB assay, as in previous studies that have demonstrated the difficulties of obtaining reliable data for these activities in other engineered microbes [18]. This result is in keeping with the observations above that 1-butanol was not produced by these strains. Ter activities were measured in crude 


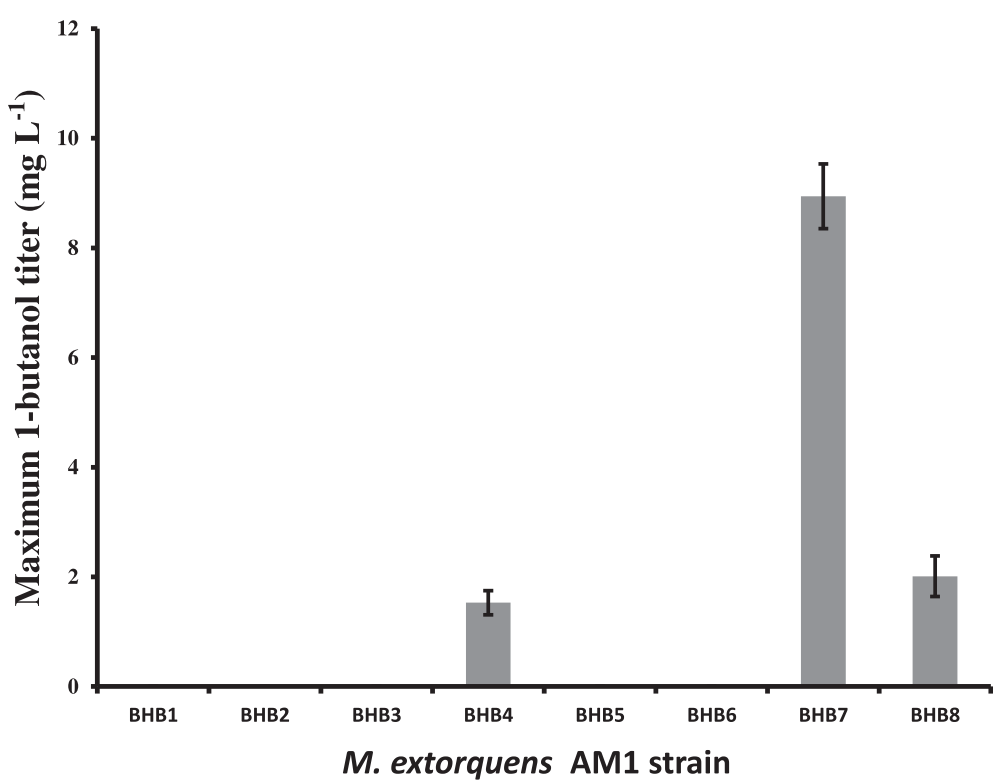

Figure 2 Maximum 1-butanol titers of engineered strains. Cells were grown in minimal medium with 20 mM ethylamine in shake flasks for 4 days. Error bars represent standard deviation of triplicate experiments.

extracts of the engineered strains, and their levels were comparable to the levels of $\mathrm{Ccr}$ in extracts of wild-type M. extorquens AM1 [36]. Since the native enzyme Ccr competes with Ter for crotonyl-CoA, both the velocity and the $\mathrm{Km}$ are important parameters. The reported $\mathrm{Km}$ value for Ter with respect to crotonyl-CoA is $2.7 \mu \mathrm{M}$, which is 100 times lower than that of the native Ccr $(400 \mu \mathrm{M})$, and the $\mathrm{Km}$ value of a recombinant Ter in E. coli with respect to crotonyl-CoA is yet five times lower than that of Ccr $[19,37,38]$. Therefore, Ter likely generates sufficient metabolic driving force toward the 1-butanol synthesis pathway, supported by the results showing that the Ter-containing strains generate detectable 1-butanol.

\section{In vitro isotopic tracing through reactions in the 1-butanol pathway}

In order to assess overall function of the entire 1-butanol synthetic pathway, assays were carried out using cell extracts of ethylamine-grown wild-type and BHB7 strains incubated with ${ }^{13} \mathrm{C}$-labeled acetyl-CoA, NADPH, and $\mathrm{NADH}$, measuring the accumulation of labeled intermediates over time. A set of parallel experiments were conducted in which reactions were incubated for $5 \mathrm{~s}$, $30 \mathrm{~s}$, and $5 \mathrm{~min}$ before quenching and mass spectrometry analysis. Through measurements of the ${ }^{13} \mathrm{C}$ isotopomer labeling patterns, we identified four of the five predicted labeled intermediates (all except for crotonyl-CoA) in the 1-butanol-producing pathway (Figure 3).

Labeled acetyl-CoA, acetoacetyl-CoA, and 3-hydroxybutyryl-CoA were detected in similar amounts for the wild type and the BHB7 strain during the time course. Crotonyl-CoA was not detected, even though the precursor to crotonyl-CoA, 3-hydroxybutyryl-CoA, and the downstream metabolite butyryl-CoA were both detected. Previous studies reported the difficulties of quantifying crotonyl-CoA in vivo because of the small pool size $[15,28]$. Small amounts of butyryl-CoA were detected in the assays of the wild type, but the amount was 13- to 43-fold higher in the BHB7 strain. This result is consistent with a previous report that a small amount of butyryl-CoA is detected in cellular extracts of M. extorquens AM1 grown on ethylamine [39]. The pathway for butyryl-CoA synthesis in wild-type $M$. extorquens AM1 is not known. It could be a side product of the native

Table 2 Enzymatic activities of Ter and AdhE2 in M. extorquens AM1 strains

\begin{tabular}{|c|c|c|c|c|c|c|}
\hline \multirow{2}{*}{$\begin{array}{l}\text { Enzyme } \\
\text { (nmol/min/mg) }\end{array}$} & \multicolumn{6}{|l|}{ Strain } \\
\hline & wild type & BHB4 & BHB5 & BHB6 & BHB7 & BHB8 \\
\hline Ter & $9.5 \pm 3.3$ & $59.1 \pm 19.8$ & $41.5 \pm 13.6$ & $52.1 \pm 12.2$ & $62 \pm 5.61$ & $89.6 \pm 22.3$ \\
\hline AdhE2 $^{*}$ & $17.5 \pm 1.4$ & $21.8 \pm 5.4$ & $24.3 \pm 3.2$ & $28.2 \pm 11.8$ & $31.3 \pm 4.8$ & $18.6 \pm 2.5$ \\
\hline AdhE2 $^{\#}$ & $1.3 \pm 0.4$ & $7.5 \pm 2.2$ & $7.4 \pm 1.2$ & $6.9 \pm 1.8$ & $11.9 \pm 0.6$ & $1.6 \pm 0.7$ \\
\hline
\end{tabular}




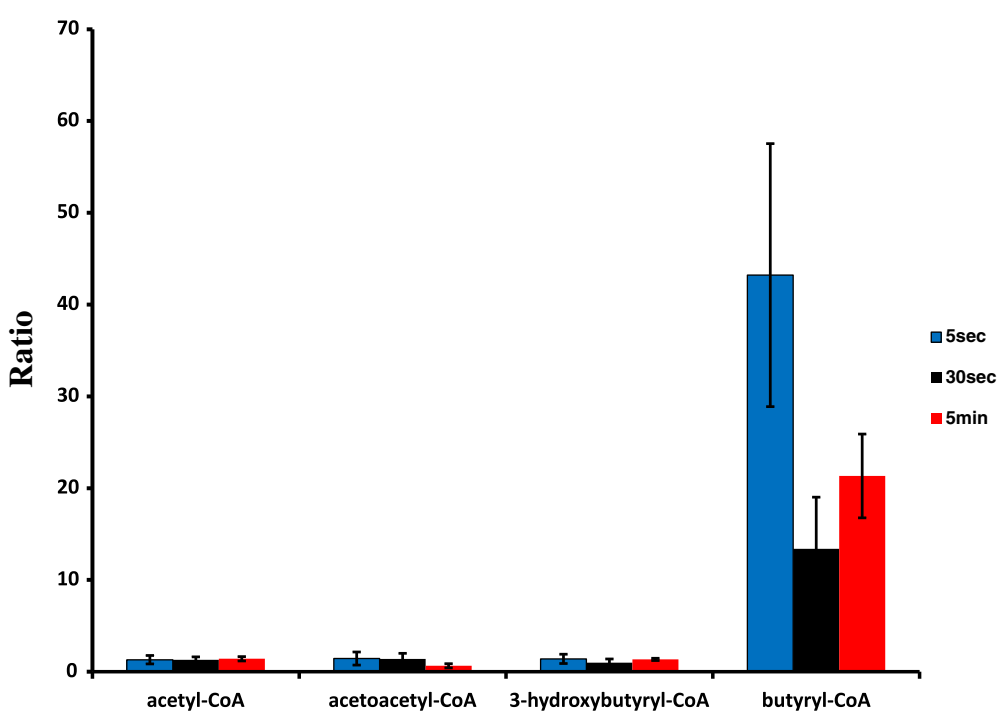

Figure 3 In vitro ${ }^{13}$ C-based metabolic flux analysis of wild-type and BHB7 strain. Results are ratios of peak volumes in BHB7 cell extracts compared to wild type. The extracts of ethylamine-grown cells were fed with ${ }^{13} \mathrm{C}$-labeled acetyl-CoA and radiolabeled metabolites were measured at different reaction times: $5 \mathrm{~s}$ (blue), $30 \mathrm{~s}$ (black), and $5 \mathrm{~min}$ (red).

reductive carboxylation reaction to form ethylmalonyl-CoA, or it could be generated from isobutyryl-CoA via valine synthesis, or from metabolism of even-numbered fatty acids $[37,40]$. However, regardless of the source, the rate of butyryl-CoA synthesis in the wild type is very low. The significant increase of labeled butyryl-CoA in strain BHB7 is consistent with the enzymatic function of Ter in vitro and shows that carbon flux to butyryl-CoA occurred via the 1-butanol synthetic pathway. In addition, accumulation of butyryl-CoA suggests that increasing the efficiency of AdhE2 might improve the system.

\section{In vivo intermediates of the 1-butanol pathway}

In order to identify potential bottlenecks of the 1-butanol pathway, we investigated in vivo intermediates by LC-MS. In these samples, four of the five intermediates were detected in cell extracts of the wild-type and BHB7 strains, all except for acetoacetyl-CoA, which either has a small pool size or is not stable in sample pretreatment. The pool sizes from BHB7 of acetyl-CoA and 3-hydroxybutyryl-CoA do not have significant differences compared to the wild type, suggesting that upstream metabolic fluxes were not significantly affected by the introduction of the 1-butanol pathway (Figure 4). However, the pools of both crotonylCoA and butyryl-CoA are more than 10-fold lower in strain BHB7 than in the wild-type strain, presumably due to the depletion of crotonyl-CoA by Ter in the BHB7 strain. The result is expected, since the accumulated butyryl-CoA could not be incorporated by central metabolic pathways in the wild-type strain but can be removed by AdhE2 for 1-butanol synthesis in strain BHB7. These results suggest that an insufficient supply of crotonyl-CoA is a bottleneck for 1-butanol production, indicating this step as a target for further strain improvement.

\section{Optimization of strain for 1-butanol production}

One of the strategies available to adjust the crotonyl-CoA supply is to decrease crotonyl-CoA flux through the EMC pathway. Ccr competes with Ter for crotonyl-CoA directly, but $\mathrm{Ccr}$ is essential for growth of $M$. extorquens in both $\mathrm{C} 1$ and $\mathrm{C} 2$ substrates [41]. Previously, we constructed a CcrR mutant which has half the Ccr activity of the wild-type strain, but maintains the ability to grow on methanol and ethylamine, albeit at a lower growth rate [42]. The constructed plasmid that was successful in strain BHB7 was introduced into this CcrR mutant, which was tested for 1-butanol production on $\mathrm{C} 1$ and $\mathrm{C} 2$ compounds. However, this strain did not produce 1-butanol on methanol, and a decreased amount of 1-butanol was detected from cells grown on ethylamine compared to the wild type, likely because the CcrR mutant grows more slowly on ethylamine compared to the wild type (data not shown).

An alternative approach to increase the total crotonylCoA supply is to overexpress crotonase. The gene encoding crotonase in $M$. extorquens AM1 (croR) was cloned under a modified tac promoter (CCACACATTATACGAGCCGAT GATTAATTGTCAACAGCTCA TTTCAGATTTCTT), which was then assembled with the 1-butanol operon (Pmeta1_3616::adhE2::ter) in BHB7 to create a new strain, BHB9. A threefold increase in CroR activity was found in the extract of BHB9 compared to BHB7 $\left(0.14 \mathrm{U} \mathrm{mg}^{-1}\right.$ protein versus $0.05 \mathrm{U} \mathrm{mg}^{-1}$ protein, respectively). When growing on ethylamine, this strain produced $50 \%$ more 1-butanol (maximum 1-butanol titer $=13.6 \mathrm{mg} \mathrm{l}^{-1}$ ) and 


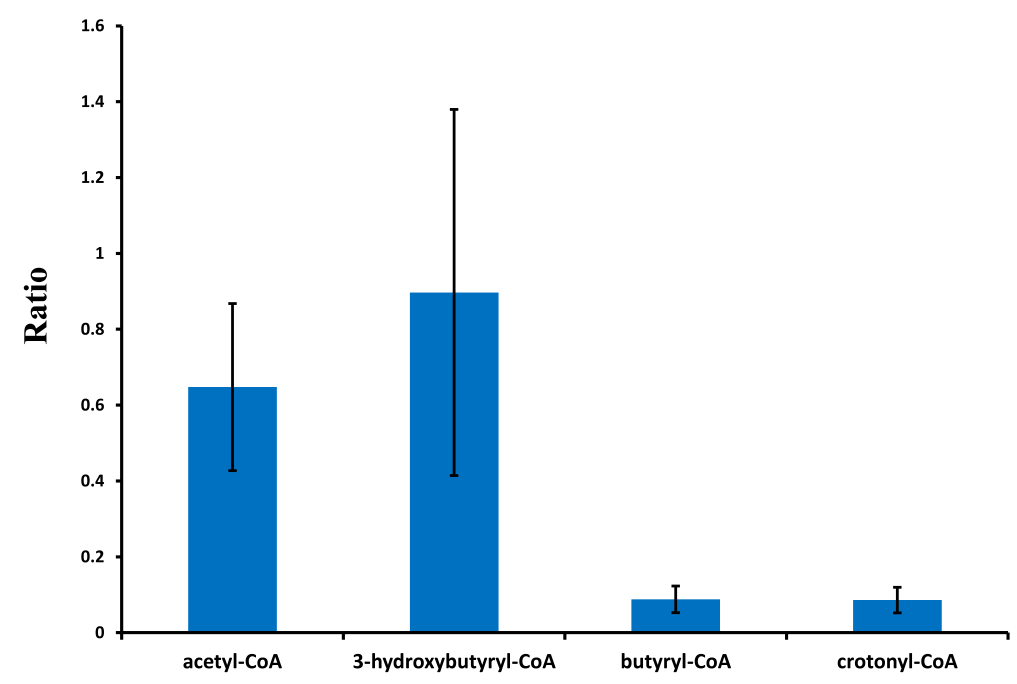

Figure 4 Comparison of in vivo 1-butanol pathway intermediates in wild type and strain BHB7. Samples were collected from ethylamine-grown cells at mid-exponential phase. Results are ratios of peak volumes in BHB7 samples compared to wild type.

no growth defect compared to BHB7 (Figure 5). These results suggest that higher flux via CroR contributes to the increased production of 1-butanol. The 1-butanol mainly accumulated in the exponential phase.

\section{1-butanol tolerance of selected mutants}

1-butanol toxicity to microorganisms is one of the important factors that limit industrial production potential of this next generation biofuel. Therefore, the growth rates of $M$. extorquens AM1 wild type in the presence of 1-butanol were investigated (Figure 6). The growth rate was calculated from growth curves monitored with different carbon sources. The results showed that 1-butanol is toxic to $M$. extorquens AM1 at $0.15 \%$ 1-butanol. In the presence of $0.15 \%$ butanol, the relative growth rates on methanol, ethylamine, and succinate were $62 \%, 58 \%$, and $72 \%$, respectively, compared to those for the medium without butanol. However, M. extorquens AM1 cannot tolerate and grow in $0.5 \%$ or higher butanol on any of these three substrates. This result suggests that further strain development for improved 1-butanol tolerance is necessary for M. extorquens AM1.

\section{Conclusions}

In this work we reported metabolic engineering of the ethylmalonyl-CoA pathway in $M$. extorquens AM1 for 1-butanol production. The 1-butanol pathway contains part of the native ethylmalonyl-CoA pathway, a transenoyl-CoA reductase from Treponema denticola, and alcohol dehydrogenase from Clostridium acetobutylicum.
A

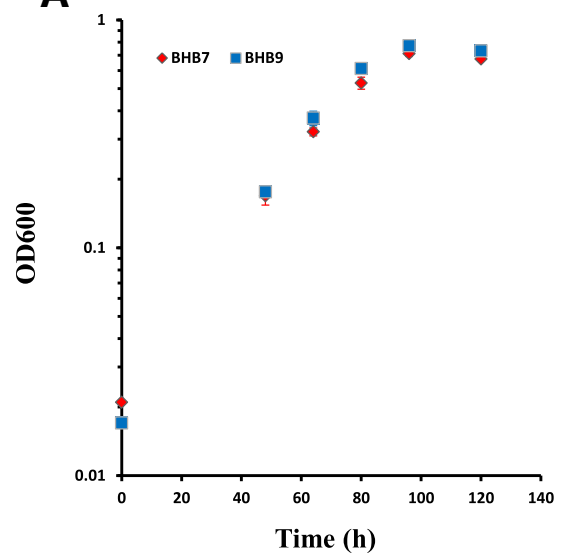

B

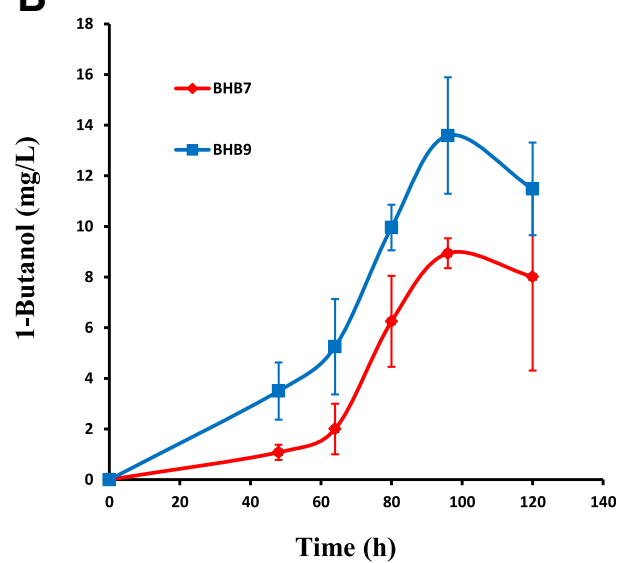

Figure 5 Effect of CroR overexpression on 1-butanol production. A) Growth of strain BHB7 (red) and BHB9 (blue). B) 1-butanol production in culture of strain BHB7 (red) and BHB9 (blue). All strains were inoculated in medium containing ethylamine (20 mM). Error bars represent standard deviation of triplicate experiments. 
The engineered strains demonstrated various maximum 1-butanol titers from cells grown on ethylamine with the highest titer of $8.94 \mathrm{mg} \mathrm{l}^{-1}$, which was further improved to $13.6 \mathrm{mg} \mathrm{l}^{-1}$ by overexpressing native crotonase. Although further strain optimization is required to make this system industrially relevant, metabolic engineering precedents exist that have resulted in similar magnitudes of increase $[43,44]$. In vitro assays suggested that the enzymatic reaction catalyzed by AdhE2 is a rate-limiting step in 1-butanol synthesis. It has been shown in cyanobacteria that substitution of AdhE2 with oxygen-insensitive butyryl-CoA reductase improves butanol production [45]. Since M. extorquens AM1 is an obligate aerobe [46], a similar strategy combined with other efforts such as increasing the reducing power and acetyl-CoA supply can be harnessed to improve 1-butanol production in the future. This study demonstrates that metabolic intermediates such as crotonyl-CoA can be used as substrates of a heterologous engineered pathway to produce value-added chemicals, setting up the first example to develop $M$. extorquens AM1 into a platform for production of biofuels.

\section{Methods}

\section{Reagents}

All chemicals including metabolite standards were purchased from Sigma-Aldrich (St. Louis, MO). Restriction enzymes, Phusion DNA polymerase, ligases, and the Gibson Assembly Master Mix kits were supplied by New England Biolabs (Ipswich, MA). Media components were purchased from commercial resources. The BCA kit for protein measurement was purchased from Thermo Scientific (Waltham, MA).

\section{Strains, medium, and growth conditions}

The strains used in this study are listed in Table 1. Escherichia coli strains Top 10 and S17-1 were cultivated at $37^{\circ} \mathrm{C}$ in Luria-Bertani medium. M. extorquens AM1 was routinely cultured in the minimal medium described previously [16] with one of the following substrates: succinate $(20 \mathrm{mM})$, methanol $(125 \mathrm{mM})$, or ethylamine (20 mM). For triparental or biparental matings between E. coli and $M$. extorquens AM1, Difco nutrient broth supplemented with Difco BiTek agar (1.5\% [wt/vol]) was used. Antibiotics were supplied at concentrations as follows: tetracycline (Tet) $20 \mu \mathrm{g} \mathrm{ml} \mathrm{m}^{-1}$, kanamycin (Km), $50 \mu \mathrm{g} \mathrm{ml} l^{-1}$, ampicillin (Amp), $100 \mu \mathrm{g} \mathrm{ml}^{-1}$, and rifamycin (Rf), $50 \mu \mathrm{g} \mathrm{m} \mathrm{m}^{-1} .1 \mathrm{mM}$ of glyoxylate was used for tests for 1-butanol production from cells grown on methanol plus glyoxylate.

Growth curves and 1-butanol production assessments were carried out in biological triplicates. Tested $M$. extorquens AM1 strains were subcultured $(0.5 \mathrm{ml})$ from tubes into $50 \mathrm{ml}$ of minimal medium in 250-ml flasks containing the appropriate carbon source, then inoculated at $30^{\circ} \mathrm{C}$ on shakers at $200 \mathrm{rpm}$.

To assess the growth rates in the presence of 1-butanol, cultures of $M$. extorquens AM1 wild type were grown to middle exponential phase in $5 \mathrm{ml}$ minimal medium at $30^{\circ} \mathrm{C} .0 .5 \mathrm{ml}$ of each culture was distributed into $50 \mathrm{ml}$ fresh medium in 250-ml screw-capped flasks containing

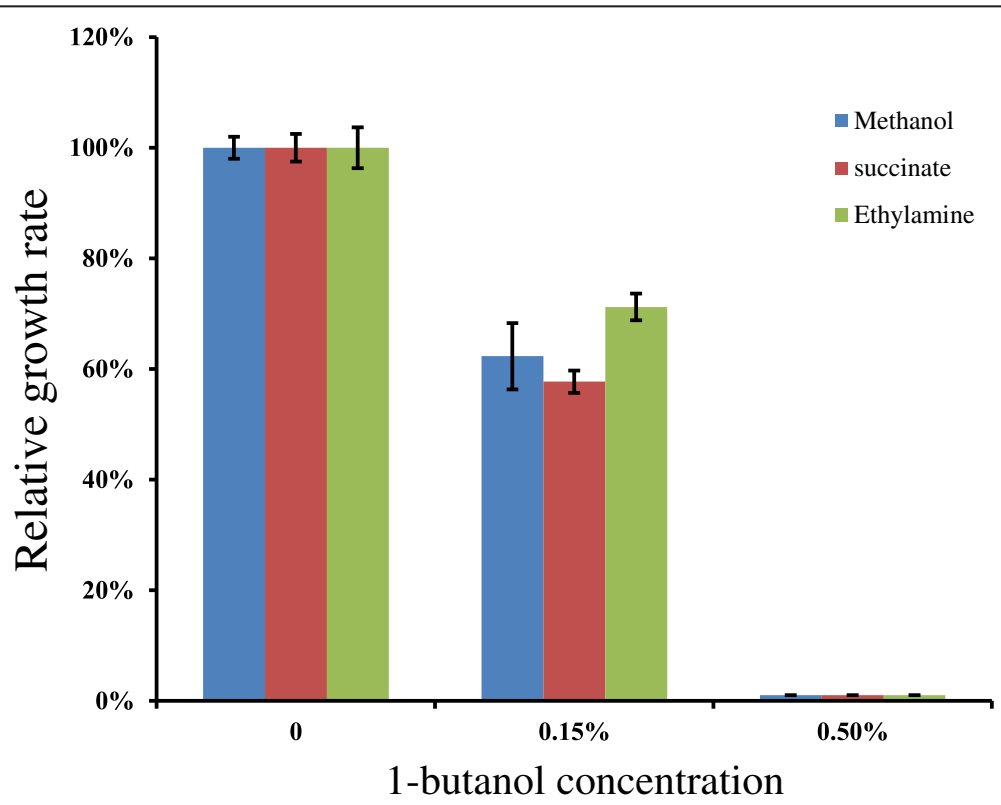

Figure 6 Growth rates in butanol relative to medium without butanol of wild-type M. extorquens AM1. Cells were grown on one of the following substrates: methanol (blue), succinate (red), or ethylamine (green). Triplicate experiments were performed for each individual measurement. 
appropriate amounts of 1-butanol, and the growth was followed over time.

\section{DNA manipulations}

The protein sequences of butyryl-CoA dehydrogenase with an affiliated electron transfer flavoprotein (Bcd and EtfAB), alcohol dehydrogenase (AdhE2), and NADH-dependent crotonyl-CoA reductase (Ter) were retrieved from GenBank with the following accession numbers: Bcd and EtfAB, [GenBank:U17110.1], AdhE2, [GenBank:AF321779.1], Ter, [GenBank:AE017248.1]. Genes coding for these enzymes were synthesized into the vector pUC57 (Genescript, NJ, USA) with codon usage optimized for expression in M. extorquens AM1 in which the codon usage frequency was calculated by counting codon frequency in a list of ORFs associated with the 129 highly expressed genes in both methanol-grown and succinate-grown $M$. extorquens AM1 cells. The gene encoding crotonase $(c r o R)$ was cloned from $M$. extorquens AM1 genomic DNA. Standard restriction enzyme digestion and ligation techniques were used to construct plasmids except for the one used to create strain BHB9, which was constructed via Gibson Assembly. Genes were PCR amplified with Phusion polymerase and assembled into the XbaI-BamHI and KpnI-EcoRI restriction sites of plasmids with different promoter regions. Four promoters were tested, one high ( $m x a F$ promoter of pCM80; [22]), one low (lac promoter of pCM62; [25]), and two in between chosen based on published microarray data results (Pmeta1_3616 promoter of pAP775 and Pmeta1_002 promoter of pAP776; [23]). The expression vectors pAP775 and pAP776 were designed and constructed by replacing the lac promoter with the putative promoter region upstream of Gene meta1_3616 and Gene meta1_002, respectively. The relative expression of the promoters was PmxaF $>>$ Pmeta1_3616 $>$ Pmeta1_002 $>$ Plac, based on relative microarray expression data. For plasmid construction of strain BHB9, the croR fragment was first assembled with a pHC61 vector fragment (under a tac promoter [47]), and the new construct was used as a vector fragment to be assembled with the region of Pmeta1_3616::adhE2::ter.

\section{Enzyme assays}

$M$. extorquens AM1 cells were harvested at midexponential phase and then resuspended in the appropriate buffer as described below for each assay. Crude cell extracts were obtained by passing the cells through a French pressure cell at $1.2 \times 10^{8} \mathrm{~Pa}$ and clarification by 15 min centrifugation at $15,000 \times \mathrm{g}$ at $4^{\circ} \mathrm{C}$. Protein concentrations were determined by the $\mathrm{BCA}$ assay using bovine serum albumin as a standard according to the instructions of the supplier. All assays were conducted at $25^{\circ} \mathrm{C}$.
Trans-2-enoyl-CoA reductase (Ter) assay

A standard spectrophotometric assay for Ter activity was performed as described by monitoring the oxidation of $\mathrm{NADH}$ at $340 \mathrm{~nm}$ [48]. The assay mixture contained $0.5 \mathrm{mM}$ crotonyl-CoA and $0.2 \mathrm{mM} \mathrm{NADH}$ in $0.1 \mathrm{M}$ potassium phosphate buffer $(\mathrm{pH} 7.5)$. The reaction was initiated by the addition of cell extracts.

\section{Butanol dehydrogenase (AdhE2) assay}

The butanol dehydrogenase activity was measured in the reverse direction as described by monitoring the increase in absorbance at $340 \mathrm{~nm}$ [49]. The assay mixture contained 19.6 mM butanol, 0.39 mM NAD+, and $78.5 \mathrm{mM}$ semicarbazide hydrochloride in $68.8 \mathrm{mM}$ Tris- $\mathrm{HCl}, \mathrm{pH}$ 7.8. The reaction was initiated by the addition of cell extracts.

\section{Butyraldehyde dehydrogenase (AdhE2) assay}

The butyraldehyde dehydrogenase activity was measured by monitoring the oxidation of NADH at $340 \mathrm{~nm}$. The assay mixture contained $200 \mu \mathrm{M}$ butyryl-CoA, $0.4 \mathrm{mM}$ $\mathrm{NADH}$, and $5 \mathrm{mM}$ dithiothreitol in $0.1 \mathrm{M}$ potassium phosphate buffer ( $\mathrm{pH} 7.5$ ). The reaction was initiated by the addition of butyryl-CoA.

\section{Crotonase (CroR) assay}

The CroR assay was conducted by monitoring the decrease of absorbance at $263 \mathrm{~nm}$, corresponding to hydration of the double bond in crotonyl-CoA. The assay mixture contained $0.15 \mathrm{mM}$ crotonyl-CoA in $0.1 \mathrm{M}$ Tris- $\mathrm{HCl}$ buffer, $\mathrm{pH}$ 7.6. The reaction was initiated by the addition of crotonyl-CoA.

\section{1-butanol quantification}

$10 \mathrm{ml}$ of culture samples were centrifuged for $10 \mathrm{~min}$ at 5,000 rpm. $2 \mathrm{ml}$ ethyl acetate was added to the supernatant. After the addition of $50 \mathrm{mg} \mathrm{l}^{-1}$ isobutanol as an internal standard, the mixture was vortexed for $1 \mathrm{~min}$ and then centrifuged for $10 \mathrm{~min}$ at 5,000 rpm to separate the aqueous phase and ethyl acetate. The recovered ethyl acetate was analyzed by an HP 6890 gas chromatograph equipped with a Model 19091 s-433 HP-5MS column (Agilent) and a single quadrupole mass spectrometer. Helium with 7.64 psi inlet pressure was used as the carrier gas. $1-\mu \mathrm{l}$ aliquots of the samples were analyzed with the following program: Set initial temperature at $50^{\circ} \mathrm{C}$, ramped to $90^{\circ} \mathrm{C}$ at $10^{\circ} \mathrm{C} / \mathrm{min}$, ramped to $300^{\circ} \mathrm{C}$ at $45^{\circ} \mathrm{C} / \mathrm{min}$, maintained at $300^{\circ} \mathrm{C}$ for $1 \mathrm{~min}$. A standard curve was derived from measurements of 1-butanol aqueous solutions (2 $\mathrm{mg} \mathrm{l}^{-1}, 5 \mathrm{mg} \mathrm{l}^{-1}$, $10 \mathrm{mg} \mathrm{l}^{-1}, 25 \mathrm{mg} \mathrm{l}^{-1}$, $50 \mathrm{mg} \mathrm{l}^{-1}$ ). The ion source temperature was set to $250^{\circ} \mathrm{C}$. Mass spectra were collected at $\mathrm{m} / \mathrm{z} 41$ and $\mathrm{m} / \mathrm{z} 56$ with a 2.2-min solvent delay. The peaks were analyzed using Agilent ChemStation software. 


\section{CoA derivative analysis}

For in vitro demonstration of the synthetic pathway, cell extracts were prepared in the same way as for enzyme assays. The reaction mixture contained $0.7 \mathrm{mM}{ }^{13} \mathrm{C}$-labeled acetyl-CoA, $0.5 \mathrm{mM} \mathrm{NADPH}, 0.23 \mathrm{mM} \mathrm{NADH}$, and $0.1 \mathrm{mM}$ Tris- $\mathrm{HCl}$ buffer, $\mathrm{pH}$ 7.5. The reaction was initiated by the addition of cell extracts and quenched by $3 \times$ volume acetonitrile at $10 \mathrm{~s}, 30 \mathrm{~s}$, or $5 \mathrm{~min}$. The mixture was centrifuged for $10 \mathrm{~min}$ at $14,000 \mathrm{rpm}$ after incubation in a $-20^{\circ} \mathrm{C}$ freezer for $1 \mathrm{~h}$. The supernatant was diluted with doubledistilled water $\left(\mathrm{ddH}_{2} \mathrm{O}\right)$ to $10 \%$ acetonitrile and frozen in liquid nitrogen before lyophilization. Each lyophilized sample

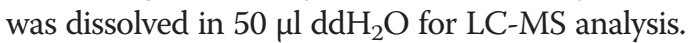

For in vivo metabolite measurements, the samples for the determination of extracellular metabolites were collected from cultures when the cells had reached an OD600 of 0.5. $10 \mathrm{ml}$ of cell culture was filtered using a Millipore membrane $(0.22 \mu \mathrm{m})$, and the membranes were then washed with 3 to $5 \mathrm{ml}$ ice-cold quench buffer (21 $\mathrm{mM}$ ammonium formate, $0.17 \%$ (v:v) formic acid, and 25\% (v:v) ethanol). The cells were broken by passing them through a French pressure cell at $1.2 \times 10^{8} \mathrm{~Pa}$ twice. The effluent was diluted by $\mathrm{dd}_{2} \mathrm{O}$ to the total volume of $30 \mathrm{ml}$. The mixture was then lyophilized to obtain LC-MS samples which

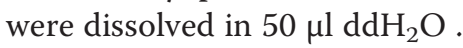

Both in vitro and in vivo metabolites were analyzed by a Waters Xevo LC-MS system consisting of an Acquity UPLC system and a Xevo triple quadrupole mass spectrometer (Milford, MA). The LC conditions for the CSH-C18 column (130 ̊, $1.7 \mu \mathrm{m}, 2.1 \mathrm{~mm} \times 100 \mathrm{~mm})$ were as follows: mobile phase A consisted of $25 \mathrm{mM}$ amonium acetate, $2 \%$ acetic acid (v:v), $1 \%$ formic acid (v:v) in water; mobile phase $\mathrm{B}$ consisted of $2 \%$ acetic acid (v:v), $1 \%$ formic acid (v:v) in acetonitrile. Initial $A=100 \%$, set $A=80 \%$ at $2.5 \mathrm{~min}, \mathrm{~A}=55 \%$ at $5 \mathrm{~min}, \mathrm{~A}=5 \%$ at $6 \mathrm{~min}, \mathrm{~A}=100 \%$ at $7 \mathrm{~min}, \mathrm{~A}=100 \%$ at $8 \mathrm{~min}$. The flow rate was $0.3 \mathrm{ml} \mathrm{min}^{-1}$. The MS was operated in the method described before [39] with minor modifications. All data were analyzed using the MassLynx QuanLynx Application Manager software.

\section{Abbreviations \\ AdhE1: bifunctional aldehyde/alcohol dehydrogenase; AdhE2: bifunctional aldehyde/alcohol dehydrogenase; ATP: adenosine triphosphate; BCA: bicinchoninic acid assay; Bcd and EtfAB: butyryl-CoA dehydrogenase and electron transferring flavoprotein; Ccr: crotonyl-CoA carboxylase/ reductase; CroR: crotonase; EMC: ethylmalonyl-CoA; NADH: nicotinamide adenine dinucleotide; NADPH: nicotinamide adenine dinucleotide phosphate; ORF: open reading frame; PhaA: $\beta$-ketothiolase; PhaB: acetoacetyl-CoA reductase; PQQ: pyrroloquinoline quinone; TCA: tricarboxylic acid; Ter: trans-2-enoyl-CoA reductase.}

\section{Competing interests}

The authors declare that they have no competing interests.

\section{Authors' contributions}

$\mathrm{BH}$ and MEL conceived and designed the project. $\mathrm{BH}$ performed the experiments. $\mathrm{BH}$ and MEL interpreted the data. Both authors contributed to the preparation of the manuscript. Both authors read and approved the final manuscript.

\section{Acknowledgements}

We thank Mila Chistoserdova, N. Cecilia Martinez-Gomez, Aaron Puri, and Nathan Good for helpful comments on the manuscript; Alexander Palmer for the construction of the PAP plasmids; and Yanfen Fu for assistance with the mass spectroscopy analysis.

\section{Author details}

${ }^{1}$ Department of Chemical Engineering, University of Washington, Seattle, WA, USA. ${ }^{2}$ Department of Microbiology, University of Washington, Seattle, WA 98195-1750, USA.

Received: 17 April 2014 Accepted: 3 October 2014

Published online: 21 October 2014

\section{References}

1. Swana J, Yang Y, Behnam M, Thompson R: An analysis of net energy production and feedstock availability for biobutanol and bioethanol. Bioresour Technol 2011, 102:2112-2117.

2. Pfromm PH, Amanor-Boadu V, Nelson R, Vadlani P, Madl R: Bio-butanol vs. bio-ethanol: a technical and economic assessment for corn and switchgrass fermented by yeast or Clostridium acetobutylicum. Biomass and Bioenergy 2010, 34:515-524.

3. Dürre P: Fermentative butanol production: bulk chemical and biofuel. Ann N Y Acad Sci 2008, 1125:353-362.

4. Dürre P: Biobutanol: an attractive biofuel. Biotechnol J 2007, 2:1525-1534.

5. Lütke-Eversloh T: Application of new metabolic engineering tools for Clostridium acetobutylicum. Appl Microbiol Biotechnol 2014, 98:5823-5837.

6. Kuehne S, Minton NP: ClosTron-mediated engineering of Clostridium. Bioengineered 2012, 3:247-254.

7. Branduardi $P$, de Ferra F, Longo V, Porro D: Microbial $n$-butanol production from Clostridia to non-Clostridial hosts. Eng Life Sci 2014, 14:16-26.

8. Atsumi S, Cann AF, Connor MR, Shen CR, Smith KM, Brynildsen MP, Chou KJY, Hanai T, Liao JC: Metabolic engineering of Escherichia coli for 1-butanol production. Metab Eng 2008, 10:305-311.

9. Steen EJ, Chan R, Prasad N, Myers S, Petzold CJ, Redding A, Ouellet M, Keasling JD: Metabolic engineering of Saccharomyces cerevisiae for the production of n-butanol. Microb Cell Fact 2008, 7:36.

10. Nielsen DR, Leonard E, Yoon S-H, Tseng H-C, Yuan C, Prather KL: Engineering alternative butanol production platforms in heterologous bacteria. Metab Eng 2009, 11:262-273.

11. Berezina OV, Zakharova NV, Brandt A, Yarotsky SV, Schwarz WH, Zverlov W: Reconstructing the clostridial n-butanol metabolic pathway in Lactobacillus brevis. Appl Microbiol Biotechnol 2010, 87:635-646.

12. Saavalainen $P$, Keiski RL: Biobutanol as a potential sustainable biofuel assessment of lignocellulosic and waste-based feedstocks. JSDEWES 2013, 1:58-77.

13. Conrado RJ, Gonzalez R: Envisioning the bioconversion of methane to liquid fuels. Science 2014, 343:621-623.

14. Schrader J, Schilling M, Holtmann D, Sell D, Filho MV, Marx A, Vorholt JA: Methanol-based industrial biotechnology: current status and future perspectives of methylotrophic bacteria. Trends Biotechnol 2009, 27:107-115.

15. Peyraud R, Kiefer P, Christen P, Massou S, Portais JC, Vorholt JA: Demonstration of the ethylmalonyl-CoA pathway by using ${ }^{13} \mathrm{C}$ metabolomics. Proc Natl Acad Sci U S A 2009, 106:4846-4851.

16. Schneider K, Peyraud R, Kiefer P, Christen P, Delmotte N, Massou S, Portais JC, Vorholt JA: The ethylmalonyl-CoA pathway is used in place of the glyoxylate cycle by Methylobacterium extorquens AM1 during growth on acetate. J Biol Chem 2012, 287:757-766.

17. Peyraud R, Schneider K, Kiefer P, Massou S, Vorholt JA, Portais JC: Genome-scale reconstruction and system level investigation of the metabolic network of Methylobacterium extorquens AM1. BMC Syst Biol 2011, 5:189.

18. Boynton ZL, Bennett GN, Rudolph FB: Cloning, sequencing, and expression of clustered genes encoding beta-hydroxybutyryl-coenzyme A (CoA) dehydrogenase, crotonase, and butyryl-CoA dehydrogenase from Clostridium acetobutylicum ATCC 824. J Bacteriol 1996, 178:3015-3024.

19. Tucci S, Martin W: A novel prokaryotic trans-2-enoyl-CoA reductase from the spirochete Treponema denticola. FEBS Lett 2007, 581:1561-1566.

20. Papoutsakis ET: Engineering solventogenic clostridia. Curr Opin Biotechnol 2008, 19:420-429.

21. Fontaine L, Meynial-salles I, Girbal L, Yang X, Croux C, Soucaille P: Molecular characterization and transcriptional analysis of adhE2, the gene 
encoding the NADH-dependent aldehyde/alcohol dehydrogenase responsible for butanol production in alcohologenic cultures of Clostridium acetobutylicum ATCC 824. J Bacteriol 2002, 184:821-830.

22. Zhang M, FitzGerald KA, Lidstrom ME: Identification of an upstream regulatory sequence that mediates the transcription of mox genes in Methylobacterium extorquens AM1. Microbiology 2005, 151:3723-3728.

23. Skovran E, Crowther GJ, Guo X, Yang S, Lidstrom ME: A systems biology approach uncovers cellular strategies used by Methylobacterium extorquens $\mathrm{AM} 1$ during the switch from multi- to single-carbon growth. PLoS One 2010, 5:e14091

24. Okubo Y, Skovran E, Guo X, Sivam D, Lidstrom ME: Implementation of microarrays for Methylobacterium extorquens AM1. OMICS 2007, 11:325-340.

25. Marx CJ, Lidstrom ME: Development of improved versatile broad-host-range vectors for use in methylotrophs and other Gram-negative bacteria. Microbiology 2001, 147:2065-2075.

26. Page MD, Anthony C: Regulation of formaldehyde oxidation by the methanol dehydrogenase modifier proteins of Methylophilus methylotrophus and Pseudomonas AM1. Microbiology 1986, 132:1553-1563.

27. Morris CJ, Lidstrom ME: Cloning of a methanol-inducible moxF promoter and its analysis in moxB mutants of Methylobacterium extorquens $\mathrm{AM} 1^{\text {rif }}$ J Bacteriol 1992, 174:4444-4449.

28. Okubo Y, Yang S, Chistoserdova L, Lidstrom ME: Alternative route for glyoxylate consumption during growth on two-carbon compounds by Methylobacterium extorquens AM1.J Bacteriol 2010, 192:1813-1823.

29. Korotkova N, Chistoserdova L, Kuksa V, Lidstrom ME: Glyoxylate regeneration pathway in the methylotroph Methylobacterium extorquens AM1. J Bacteriol 2002, 184:1750-1758.

30. Lan El, Liao JC: Metabolic engineering of cyanobacteria for 1-butanol production from carbon dioxide. Metab Eng 2011, 13:353-363.

31. Shen CR, Lan El, Dekishima Y, Baez A, Cho KM, Liao JC: Driving forces enable high-titer anaerobic 1-butanol synthesis in Escherichia coli. Appl Environ Microbiol 2011, 77:2905-2915.

32. Lee SY, Park JH, Jang SH, Nielsen LK, Kim J, Jung KS: Fermentative butanol production by Clostridia. Biotechnol Bioeng 2008, 101:209-228.

33. Chistoserdova L, Crowther GJ, Vorholt JA, Skovran E, Portais JC, Lidstrom ME: Identification of a fourth formate dehydrogenase in Methylobacterium extorquens AM1 and confirmation of the essential role of formate oxidation in methylotrophy. J Bacteriol 2007, 189:9076-9081.

34. Van Dien SJ, Lidstrom ME: Stoichiometric model for evaluating the metabolic capabilities of the facultative methylotroph Methylobacterium extorquens AM1, with application to reconstruction of $\mathrm{C} 3$ and $\mathrm{C} 4$ metabolism. Biotechnol Bioeng 2002, 78:296-312.

35. Inui M, Suda M, Kimura S, Yasuda K, Suzuki H, Toda H, Yamamoto S, Okino S, Suzuki N, Yukawa H: Expression of Clostridium acetobutylicum butanol synthetic genes in Escherichia coli. Appl Microbiol Biotechnol 2008, 77:1305-1316.

36. Smejkalová H, Erb TJ, Fuchs G: Methanol assimilation in Methylobacterium extorquens AM1: demonstration of all enzymes and their regulation. PLoS One 2010, 5:e13001

37. Erb TJ, Brecht V, Fuchs G, Müller M, Alber BE: Carboxylation mechanism and stereochemistry of crotonyl-CoA carboxylase/reductase, a carboxylating enoyl-thioester reductase. Proc Natl Acad Sci U S A 2009, 106:8871-8876.

38. Bond-watts BB, Weeks AM, Chang MC: Biochemical and structural characterization of a trans-enoyl-CoA reductase from Treponema denticola. Biochemistry 2012, 51:6827-6837.

39. Yang S, Sadilek M, Synovec RE, Lidstrom ME: Liquid chromatography-tandem quadrupole mass spectrometry and comprehensive two-dimensional gas chromatography-time-of-flight mass spectrometry measurement of targeted metabolites of Methylobacterium extorquens AM1 grown on two different carbon sources. J Chromatogr A 2009, 1216:3280-3289.

40. Zhang K, Sawaya MR, Eisenberg DS, Liao JC: Expanding metabolism for biosynthesis of nonnatural alcohols. Proc Natl Acad Sci U S A 2008, 105:20653-20658.

41. Chistoserdova LV, Lidstrom ME: Molecular characterization of a chromosomal region involved in the oxidation of acetyl-CoA to glyoxylate in the isocitrate-lyase-negative methylotroph Methylobacterium extorquens AM1. Microbiology 1996, 142:1459-1468.

42. Hu B, Lidstrom M: CcrR, a TetR family transcriptional regulator, activates the transcription of a gene of the ethylmalonyl coenzyme A pathway in Methylobacterium extorquens AM1.J Bacteriol 2012, 194:2802-2808.
43. Lan El, Liao JC: ATP drives direct photosynthetic production of 1-butanol in cyanobacteria. Proc Natl Acad Sci U S A 2012, 109:6018-6023.

44. Paddon CJ, Westfall PJ, Pitera DJ, Benjamin K, Fisher K, McPhee D, Leavel MD, Tai A, Main A, Eng D, Polichuk DR, Teoh KH, Reed DW, Treynor T, Lenihan J, Fleck M, Bajad S, Dang G, Dengrove D, Diola D, Dorin G, Ellens KW, Fickes S, Galazzo J, Gaucher SP, Geistlinger T, Henry R, Hepp M, Horning $\mathrm{T}$, lqbal T, et al: High-level semi-synthetic production of the potent antimalarial artemisinin. Nature 2013, 496:528-532.

45. Lan El, Ro SY, Liao JC: Oxygen-tolerant coenzyme A-acylating aldehyde dehydrogenase facilitates efficient photosynthetic $n$-butanol biosynthesis in cyanobacteria. Energy Environ Sci 2013, 6:2672.

46. MarX CJ: Recovering from a bad start: rapid adaptation and tradeoffs to growth below a threshold density. BMC Evol Biol 2012, 12:109.

47. Lee M-C, Chou H-H, Marx CJ: Asymmetric, bimodal trade-offs during adaptation of methylobacterium to distinct growth substrates. Evolution 2009, 63:2816-2830.

48. Bond-watts BB, Bellerose RJ, Chang MCY: Enzyme mechanism as a kinetic control element for designing synthetic biofuel pathways. Nat Chem Biol 2011, 7:3-8.

49. Hartmanis MG, Gatenbeck S: Intermediary metabolism in Clostridium acetobutylicum: levels of enzymes involved in the formation of acetate and butyrate. Appl Environ Microbiol 1984, 47:1277-1283.

doi:10.1186/s13068-014-0156-0

Cite this article as: $\mathrm{Hu}$ and Lidstrom: Metabolic engineering of Methylobacterium extorquens AM1 for 1-butanol production. Biotechnology for Biofuels 2014 7:156.

\section{Submit your next manuscript to BioMed Central and take full advantage of:}

- Convenient online submission

- Thorough peer review

- No space constraints or color figure charges

- Immediate publication on acceptance

- Inclusion in PubMed, CAS, Scopus and Google Scholar

- Research which is freely available for redistribution 